\title{
Analysis of a Syntrophic Growth of Lactobacillus plantarum and Streptococcus faecalis
}

\author{
By N. R. KRIEG* and M. J. PELCZAR, Jun. \\ Department of Microbiology, University of Maryland, College Park, \\ Maryland, U.S.A.
}

(Received 10 October 1960)

\section{SUMMARY}

Syntrophism was demonstrated between strains of Lactobacillus plantarum and Streptococcus faecalis in a chemically defined medium which lacked phenylalanine and pteroylglutamic acid (PGA); however, the presence of $p$-aminobenzoic acid (PABA) was essential. Enumeration of the two organisms in syntrophic growth was found to be possible by the use of Trypticase soy agar $+\mathbf{0 . 2 5} \%(\mathrm{w} / \mathrm{v})$ glucose at pH $9 \cdot 0$ as a selective medium for $S$. faecalis. It was found that $S$. faecalis predominated in the early stages of the syntrophic growth, but that L. plantarum eventually predominated. Characterization by a bioautographic technique of the phenylalanine-active factor synthesized by $S$. faecalis and required by $L$. plantarum indicated that the factor was not identical with phenylalanine or shikimic acid. Microbiological assays of the culture fluid from cultures of $L$. plantarum indicated that three types of folic acid compounds were synthesized: (1) oxygen-stable compounds which supported the growth of Pediococcus cereviseae; (2) oxygen-labile compounds which supported the growth of $\boldsymbol{P}$. cereviseae; (3) oxygen-stable compounds which supported the growth of $S$. faecalis but not of $P$. cereviseae.

\section{INTRODUCTION}

Syntrophic growth (mutual feeding) of Lactobacillus plantarum strain 17-5 with Streptococcus faecalis strain $R$ was first reported by Nurmikko (1954). In a chemically defined medium which lacked phenylalanine (required by $L$. plantarum) and pteroylglutamic acid (PGA; required by $S$. faecalis), neither organism grew alone; but in mixed culture, abundant growth of both organisms occurred. It was suggested that this syntrophic growth occurred because each organism synthesized the omitted growth factor required by the other, i.e. phenylalanine was synthesized by $S$. faecalis and PGA was synthesized by $L$. plantarum. Koft \& Morrison (1956) reported much the same phenomenon. Judge (1958) studied this and other examples of syntrophism, and analysed the culture filtrates of $S$. faecalis to determine the nature of the phenylalanine-active substance synthesized and present in the culture filtrate. A one-dimensional ascending chromatographic technique was used, and the presence of a spot, corresponding to the position of phenylalanine, was demonstrated with a chemical indicator.

* Present address : Department of Bacteriology, Virginia Polytechnic Institute, Blacksburg, Virginia, U.S.A. 
The purpose of the present investigation was directed toward a further analysis of this system of syntrophic growth, and can be divided into two parts: (1) population aspects of the syntrophism, (2) the nature of the factor(s) synthesized by each of the two organisms involved in the mutual growth promotion.

\section{METHODS}

Organisms. The two organisms used in the syntrophic growth experiments were Lactobacillus plantarum strain 17-5 (American Type Culture Collection, Washington 7, D.C., U.S.A., ATCC No. 8014) and Streptococcus faecalis strain R (ATCC No. 8043). For the microbiological assay of folic acid-like compounds, the following organisms were used, in addition to $S$. faecalis, namely, Lactobacillus casei (ATCC No.7469) and Pediococcus cereviseae (ATCC No. 8081). All cultures were maintained in stabs of Micro assay culture agar (BBL; Baltimore Biological Laboratory, Inc., Baltimore 18, Maryland, U.S.A.) in the refrigerator: new transfers were made every 2-3 weeks.

Media. The defined medium used was that described by Nurmikko (1954) except for variations in the phenylalanine, $p$-amino benzoic acid (PABA) and PGA content. The composition of this medium is also described in an earlier report from this laboratory (Krieg \& Pelczar, 1960). The term basal medium, as used here, refers to Nurmikko's medium minus $p$-aminobenzoic acid (PABA), pteroylglutamic acid (PGA) and phenylalanine.

Analysis of syntrophic growth. Lactobacillus plantarum and Streptococcus faecalis were grown separately in the basal defined medium supplemented with $200 \mu \mathrm{g}$. phenylalanine, $0 \cdot 2 \mu \mathrm{g}$. PABA and $0 \cdot 01 \mu \mathrm{g}$. PGA $/ \mathrm{ml}$. After incubation for $18 \mathrm{hr}$. the cultures were centrifuged, washed twice with sterile physiological saline, and then resuspended in sterile distilled water to an optical density of 0.150 at $4.20 \mathrm{~m} \mu$ as measured in a $16 \mathrm{~mm}$. cuvette with a Bausch and Lomb Spectronic 20 colorimeter. These suspensions were then further diluted $1 / 10$ in sterile distilled water. These dilutions were used as inocula $(0.1 \mathrm{ml}$./tube containing $5.0 \mathrm{ml}$. medium) and, respectively, contained about $6.5 \times 10^{6}$ viable organisms of $S$. faecalis, or $7.5 \times 10^{6}$ of L. plantarum $/ \mathrm{ml}$. The scheme of inoculation (single cultures and cultures in admixture) to assess the effect of PGA, PABA and phenylalanine is evident from Fig. 1. It can be seen that the variations in media concerned the phenylalanine, PABA, and PGA content, and variations in inocula concerned the use of single cultures of each organism or both organisms inoculated together into the same medium. All incubations were at $35^{\circ}$, for various times (Fig. 1) in an air incubator. Growth was measured turbidimetrically at $600 \mathrm{~m} \mu$ in $16 \mathrm{~mm}$. optically matched screw-capped tubes.

In experiments where the two syntrophic organisms were enumerated separately from the mixed culture (Table 1), the inoculum was prepared as described above except that the concentrations of PABA and PGA were changed to 0.002 and $0.05 \mu \mathrm{g} . / \mathrm{ml}$., respectively. Growth was measured by three methods : direct count, colony count, turbidimetrically (Table 2).

Analysis of Lactobacillus plantarum culture medium for folic acid activity. For the production of the folic acid-like factor(s) synthesized by Lactobacillus plantarum, $5.0 \mathrm{ml}$. basal medium supplemented with $200 \mu \mathrm{g}$. phenylalanine and $0.05 \mu \mathrm{g}$. $\mathrm{PABA} / \mathrm{ml}$. were inoculated from the stock stab culture. After incubation for $24 \mathrm{hr}$., 
a second transfer was made to the same medium. After a further incubation for $18 \mathrm{hr}$., $1.0 \mathrm{ml}$. of the culture was added to $300 \mathrm{ml}$. of the same medium supplemented with an excess of PABA (1 mg./ml.). After incubation for $24 \mathrm{hr}$. the culture was divided into two portions : one portion was untreated, while ascorbic acid (10 $\mathrm{mg} . / \mathrm{ml}$.) was added to the other. Both portions were centrifuged to remove the organisms and the supernatant fluids adjusted to $\mathrm{pH} 6.7$ with a measured quantity of $2 \mathrm{~N}-\mathrm{NaOH}$. The two portions of supernatant fluid were kept frozen at $-10^{\circ}$ until assayed for folic acid activity; the portion which contained ascorbic acid was held in an atmosphere of argon.

Assays with Pediococcus cereviseae were made with the medium of Sauberlich (1949); 6.0 ml. of assay medium were used per tube. When assays were performed with the ascorbic acid-containing portion, $1.33 \mathrm{mg}$. ascorbic acid was added to each ml. of single-strength assay medium, which was adjusted to $\mathrm{pH} \mathrm{6.3.} \mathrm{Besides}$ being used for the assay, this ascorbate medium was used as a diluent for the ascorbic acid-containing portion described above. All media, after all additions to tubes, were autoclaved at $112^{\circ}$ for $5 \mathrm{~min}$. The inoculum culture consisted of the second of two consecutive serial $24 \mathrm{hr}$. broth cultures grown in the assay medium supplemented with $1 \mathrm{~m} \mu \mathrm{g}$. leucovorin/ml.; the culture was centrifuged, resuspended in $5 \mathrm{ml}$. sterile physiological saline and diluted 1/100 with sterile saline. One drop of this dilution served as inoculum for each assay tube. (Leucovorin was obtained from the Lederle Laboratories Division, American Cyanamid Company, Pearl River, New York, U.S.A., in solution in ampoules.) Incubation was for $24 \mathrm{hr}$. at $35^{\circ}$, and growth was determined turbidimetrically at $600 \mathrm{~m} \mu$. For the aerobic $\boldsymbol{P}$. cereviseae assay a second method, which yielded results almost identical with those given by the first method described, was used; this second assay was performed with the medium and method described in the Difco Manual (1953). Assays with Streptococcus faecalis were also performed by using the medium and method described in the same publication (Difco Manual, 1953). Assays performed with the ascorbic acid-containing portion were treated as described for the $\boldsymbol{P}$. cereviseae assay.

Assays with Lactobacillus case $i$ were made by using the basal medium of Nurmikko (1954); $6.0 \mathrm{ml}$. media were used per tube. The medium was autoclaved at $112^{\circ}$ for 5 min., with the glucose added before autoclaving. The inoculum was prepared like the Streptococcus faecalis inoculum. Growth was recorded turbidimetrically after incubation for $60 \mathrm{hr}$.

In each assay method, standard curves were prepared by using leucovorin, and the results were expressed in terms of leucovorin activity.

Analysis of Streptococcus faecalis culture medium for phenylalanine activity. For production of the phenylalanine-active factor synthesized by Streptococcus faecalis, $5.0 \mathrm{ml}$. of the basal medium, supplemented after autoclaving with $0.05 \mu \mathrm{g}$. PGA/ml., were inoculated from the stock stab culture. After $24 \mathrm{hr}$. a second transfer was made to the same medium, and after a further $18 \mathrm{hr}$., one loopful was transferred to $100 \mathrm{ml}$. of the same medium.

The concentration of the phenylalanine-active factor was accomplished in the following way. After incubation of Streptococcus faecalis for $24 \mathrm{hr}$. in the medium described, the organisms were removed by centrifugation, the supernatant fluid adjusted to $\mathrm{pH} 6.7$ with $2 \mathrm{~N}-\mathrm{NaOH}$ and evaporated to dryness at $60^{\circ}$ in a 11 . beaker. The residue was redissolved in a minimum quantity of water and extracted with 
$100 \mathrm{ml} .95 \%(\mathrm{v} / \mathrm{v})$ ethanol in water, followed by a second extraction with $50 \mathrm{ml}$. $95 \%(\mathrm{v} / \mathrm{v})$ ethanol. The ethanolic extract was evaporated to dryness at $60^{\circ}$, redissolved in a minimal amount of water, and preserved at $-10^{\circ}$ until assayed for activity.

Bioautography of the ethanolic extract for phenylalanine activity was accomplished in the following way, a modification of a method described by Block, Durrum \& Zweig (1958). The concentrate was applied in a band to Whatman No. 1 chromatography paper; after drying, it was developed by an ascending technique with the solvent acetone + water + urea $(60+40+0 \cdot 5 ; \mathrm{v}, \mathrm{v}, \mathrm{w})$. After drying, the paper was again developed with $0.04 \mathrm{~N}-\mathrm{HCl}$ at right angles to the first direction so that the phenylalanine-active factor was concentrated at the top of the paper. The paper was dried and then placed face down on basal medium solidified with $1.5 \%$ $(\mathrm{w} / \mathrm{v}$ ) agar and supplemented with $0.05 \mu \mathrm{g}$. PABA $/ \mathrm{ml}$., previously seeded with Lactobacillus plantarum. The paper strip was removed after $1 \mathrm{hr}$., and the plates incubated overnight.

Conventional methods of 'spotting' were tried on numerous occasions without. success, probably because of the interference with the migration of the test compounds by the high concentration of other solutes in the concentrate. Even with the present method, however, when compounds such as shikimic acid were chromatographed from pure solution, in contrast to being incorporated into the ethanolic extract concentrate, a considerable difference was noticed between the $\boldsymbol{R}_{f}$ values. When applied from pure solution, the shikimic acid migrated much further than when incorporated in concentrate. Therefore, special care was taken to avoid the comparison of $\boldsymbol{R}_{f}$ values of pure compounds with the $\boldsymbol{R}_{f}$ values of the phenylalanine-active factor in the concentrate.

\section{RESULTS}

Figure 1 presents the results obtained when various mixtures of phenylalanine, PABA and PGA were supplied for the growth of Lactobacillus plantarum and Streptococcus faecalis, when grown separately and in mixed culture. It can be seen that syntrophic growth occurred in the medium which lacked phenylalanine and PGA but was supplemented with PABA (designated by code 19 in Fig. 1). In media of the same composition (Fig. 1; codes 5 and 12), neither organism alone grew significantly during the incubation periods recorded. Syntrophic growth was not observed when PABA was not present in the medium.

Growth of Lactobacillus plantarum in the absence of phenylalanine eventually occurred upon prolonged incubation. The time at which such growth appeared was quite variable, but generally occurred within 3-5 days. Experiments wherein the size of the inoculum was decimally decreased indicated that after incubation for $120 \mathrm{hr}$., even those tubes which had been theoretically inoculated with one L. plantarum organism eventually exhibited growth in the basal medium supplemented with PABA but lacking phenylalanine. These results indicated that carry-over of phenylalanine in the inoculum or in the organisms of the inoculum was not a factor in this delayed response.

In the present study a direct microscopic counting procedure, as by using the Petroff-Hauser counting chamber, could not be used to follow the growth of each 
of the two organisms in syntrophic culture, because of the inability to distinguish microscopically in this technique the organisms of Lactobacillus plantarum from those of Streptococcus faecalis, On solid media, such as microassay culture agar (BBL), L. plantarum was found as long rods; but in the basal medium supplemented with phenylalanine and PABA, the organism occurred as short rods or cocci. In microinoculum broth (BBL) stained smears made up to $18 \mathrm{hr}$. revealed short rods or cocci; after $25 \mathrm{hr}$. they revealed predominantly long rods.

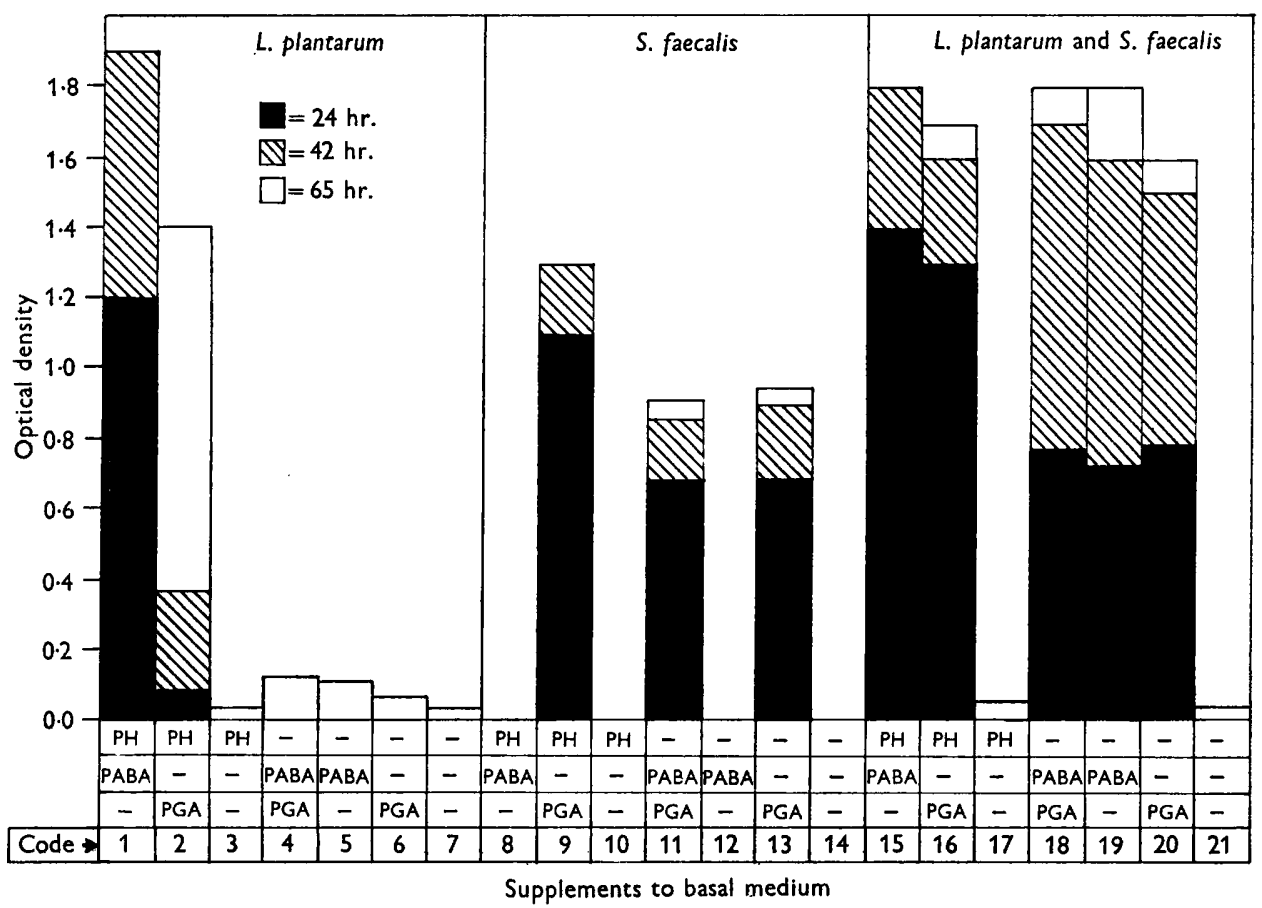

Fig. 1. The effect of phenylalanine, PABA, and PGA in the nutrition of $L$. plantarum and $S$. faecalis at $35^{\circ}$ in a chemically defined medium. The medium to which the compounds were added is the basal medium referred to in the text under Methods. PH = phenylalanine, $200 \mu \mathrm{g} . / \mathrm{ml}$; PGA $=$ pteroylglutamic acid, $0.01 \mu \mathrm{g} . / \mathrm{ml}$.; PABA $=p$ aminobenzoic acid, $0 \cdot 2 \mu \mathrm{g} . / \mathrm{ml}$. Code designations 1 through 21 identify various supplements and inocula.

Trypticase soy agar (BBL) supplemented with $0.25 \%(\mathrm{w} / \mathrm{v})$ glucose at $\mathrm{pH} \mathbf{9 \cdot 0}$ was found to serve as a selective medium for the growth of Streptococcus faecalis. The same medium at $\mathrm{pH} 7 \cdot 3$ supported the growth of both $S$. faecalis and Lactobacillus plantarum. The selective medium was used to determine the actual numbers of each organism during a period of syntrophic growth. The results (Table 1) indicate that $S$. faecalis predominated in the culture up to $26 \mathrm{hr}$, but that after $46 \mathrm{hr}$. it decreased markedly so that $L$. plantarum eventually predominated. The discrepancies apparent as between the results obtained by the three methods of enumerating the total population can be attributed to: (1) differences in size of organisms at different times; (2) the presence of non-viable, as well as viable, organisms as incubation was prolonged. 
Table 1. Growth, as measured turbidimetrically, microscopically, and by the standard plate count, of Lactobacillus plantarum and Streptococcus faecalis in pure and mixed cultures

Growth was in basal medium (see Methods) lacking phenylalanine and PGA, and supplemented with $0.002 \mu \mathrm{g}$. PABA/ml. Incubation at $35^{\circ}$.

The standard colony count for $S$. faecalis was determined by plating the syntrophic culture in Trypticase soy agar supplemented with $0.25 \%(w / v)$ glucose and adjusted to $\mathrm{pH} \mathrm{9.0.}$

The total (S. faecalis and $L$. plantarum) colony count was determined with the same medium at $\mathrm{pH} 7 \cdot 3$. The difference between the two counts represented the standard colony count for $L$. plantarum.

\begin{tabular}{|c|c|c|c|}
\hline \multirow{2}{*}{$\begin{array}{c}\text { Incubation } \\
\text { time (hr.) }\end{array}$} & \multicolumn{2}{|c|}{ Pure cultures } & \multirow[t]{2}{*}{ Both cultures } \\
\hline & S. faecalis & L. plantarum & \\
\hline & \multicolumn{3}{|c|}{ Optical density $(600 \mathrm{~m} \mu)$} \\
\hline 22 & 0.00 & 0.05 & $0 \cdot 37$ \\
\hline 46 & 0.00 & 0.05 & 1.50 \\
\hline 51 & $0 \cdot 00$ & 0.06 & $1 \cdot 70$ \\
\hline 74 & 0.00 & $1 \cdot 70$ & $1 \cdot 70$ \\
\hline \multicolumn{4}{|c|}{ Petroff-Hauser total count (cells $/ \mathrm{ml}$.) } \\
\hline 22 & - & - & $1,630,000,000$ \\
\hline 46 & - & - & $5,000,000,000$ \\
\hline 51 & - & - & $4,500,000,000$ \\
\hline 74 & - & - & $9,000,000,000$ \\
\hline \multicolumn{4}{|c|}{ Standard plate counts from syntrophic culture } \\
\hline 22 & $795,000,000$ & $\mathbf{8 5 , 0 0 0 , 0 0 0}$ & $830,000,000$ \\
\hline 46 & $30,000,000$ & $2,605,000,000$ & $2,635,000,000$ \\
\hline 51 & $<1,000,000$ & $2,694,000,000$ & $2,694,000,000$ \\
\hline 74 & $<1,000,000$ & $1,830,000,000$ & $1,830,000,000$ \\
\hline
\end{tabular}

In preliminary assays for the presence of the phenylalanine-active factor synthesized by Streptococcus faecalis, the ethanolic extract of the culture medium from a $24 \mathrm{hr}$. culture of $S$. faecalis, and the ethanol-insoluble residue remaining after this extraction, were added in different amounts to sterile paper disks; these were placed on the surface of solidified basal medium supplemented with PABA (but lacking phenylalanine), which had been previously seeded with Lactobacillus plantarum. The presence of the phenylalanine-active factor was found to reside in the ethanolic extract, as exhibited by the growth response around the corresponding disk. A bioautograph was prepared in the manner previously described, on which the ethanolic extract alone was compared with the extract to which phenylalanine had been added. It was evident that the difference in the positions of phenylalanine and of the phenylalanine factor in the extract ruled out the possibility that the factor was identical with phenylalanine. The factor was a faster moving compound than phenylalanine in the solvent system used.

A second bioautograph was prepared in which pure shikimic acid was compared with extract containing added shikimic acid. The pure shikimic acid was used to demonstrate that it was possible to obtain a 'spot' with the concentration used. However, the appearance of two 'spots' on the system when extract+shikimic acid was tested, indicated that the factor synthesized by Streptococcus faecalis was not identical with shikimic acid. The fact that shikimic acid migrated more slowly 
when incorporated in the extract, than when applied from pure solution, was also demonstrated. Nevertheless, the appearance of the shikimic acid 'spots', although different in position, clearly identified the known compound. The 'spot' of low density, that of the excreted factor, was similar in position and size to that exhibited in the preceding bioautographic experiment. The difference in positions of the two shikimic acid 'spots' may be attributed to interference with migration of the compound by the high concentration of other solutes in the extract.

The results of microbiological assays of the fractions prepared from the culture medium of Lactobacillus plantarum cultures, obtained as previously described, are presented in Table 2. The quantitative aspect of these results has significance with respect to the nature of the folic acid-like compound(s) synthesized and excreted into the medium by $L$. plantarum, in the light of recent developments in the field of folic acid metabolism. This topic is discussed below.

Table 2. Folic acid activity present in Lactobacillus plantarum culture medium after 24 $\mathrm{hr}$. growth, as assayed by Pediococcus cereviseae, Streptococcus faecalis, and Lactobacillus casei with leucovorin as reference standard

\begin{tabular}{|c|c|c|c|}
\hline \multirow[t]{2}{*}{ Organism } & Type of assay & $\begin{array}{c}\text { Sample } \\
\text { no. } 1\end{array}$ & $\begin{array}{c}\text { Sample } \\
\text { no. } 2\end{array}$ \\
\hline & & \multicolumn{2}{|c|}{$\begin{array}{l}\text { Equiv. m } \mu \mathrm{g} . \\
\text { leucovorin/ml. } \\
\text { culture fluid }\end{array}$} \\
\hline P. cereviseae & $\begin{array}{l}\text { Aerobic } \\
\text { Anaerobic* }\end{array}$ & $\begin{array}{r}3.7 \\
14 \cdot 8\end{array}$ & $\begin{array}{r}2 \cdot 9 \\
11 \cdot 6\end{array}$ \\
\hline L. casei & Aerobic & $9 \cdot 9$ & 10.2 \\
\hline S. faecalis & $\begin{array}{l}\text { Aerobic } \\
\text { Anaerobic }\end{array}$ & $\begin{array}{l}11.2 \\
24 \cdot 8\end{array}$ & $\begin{array}{l}10 \cdot 1 \\
22 \cdot 3\end{array}$ \\
\hline
\end{tabular}

* Anaerobic $=$ addition of $1.33 \mathrm{mg}$. ascorbic acid $/ \mathrm{ml}$. assay medium, with adjustment of medium to $\mathrm{pH} 6 \cdot 3$.

\section{DISCUSSION}

The failure to demonstrate syntrophic growth of Lactobacillus plantarum with Streptococcus faecalis in the absence of added PABA was in contrast to the results obtained by Nurmikko (1954) who reported syntrophic growth of the two organisms in a medium lacking PABA (designated code 21 in Fig. 1), although the growth was clearly slower than in a medium which lacked phenylalanine and PGA. Only when the purines and uracil were omitted, in addition to PABA, were the two organisms unable to grow together. In the present investigation, the medium contained the purines and uracil; but in the absence of PABA, syntrophic growth did not occur, even on prolonged incubation.

Although growth of Lactobacillus plantarum in the presence of phenylalanine, but not of PABA or PGA, did appear after prolonged incubation, within the first 3-5 days independent requirements for phenylalanine and PABA could be demonstrated. This observation is also in contrast to the results of Nurmikko (1954), who reported that the requirement for PABA could be effectively replaced by phenylalanine, that this replacement could be inhibited by sulphonamides, and that increasing concentrations of phenylalanine were effective in overcoming the 
inhibiting action of the sulphonamides. When one considers that $L$. plantarum has long been used as an assay organism for PABA in a medium containing Noritetreated acid-hydrolysed casein (Lewis, 1942), it does not appear likely that there could be a replacement of PABA by phenylalanine.

In microbiological assays involving tetrahydroPGA, it has been found that the $\mathrm{pH}$ value of the medium is important when the standard curve is based upon response to leucovorin (Donaldson \& Keresztesy, 1959). Therefore, at pH 6.3 (the pH value used in the anaerobic assays shown in Table 2), the activity of the oxygen-labile compounds assayed in the Pediococcus cereviseae system, and represented by the difference between the aerobic and anaerobic $\boldsymbol{P}$. cereviseae assays, may be much higher than is indicated, if tetrahydroPGA be assumed to be the major component of this fraction.

The assumption is made, for purposes of this discussion, that differences in $\mathrm{pH}$ value will not affect compounds other than tetrahydroPGA; otherwise, comparison becomes impossible because of the use of different $\mathrm{pH}$ values for aerobic and anaerobic assays, or because of the use of different organisms. Therefore, with this assumption, the kinds of folic acid compounds (in a general way only) can be deduced from the results of Table 2. Moreover, the concentrations of each of the different folic acid-like entities synthesized by Lactobacillus plantarum can also be determined as summarized in Table $\mathbf{3}$.

Table 3. Summary of types of folic acid-like compounds synthesized by Lactobacillus plantarum as calculated from microbiological assay data

Type of folic acid compounds

1. Oxygen-stable, for $P$. cereviseae (e.g. leucovorin)

2. Oxygen-stable and oxygen-labile, for $P$. cereviseae (e.g. leucovorin + tetrahydroPGA)

3. Oxygen-labile, for $P$. cereviseae (e.g. tetrahydroPGA)

4. Oxygen-stable, for $L$. casei (e.g. leucovorin) + PGA

5. Oxygen-stable, for L. casei but not stimulatory for $P$. cereviseae (e.g. PGA)

6. Oxygen-stable, for $\boldsymbol{S}$. faecalis (e.g. leucovorin) + PGA

7. Oxygen-stable, for $S$. faecalis but not stimulatory for $P$. cereviseae (e.g. PGA)

8. Oxygen-stable and oxygen-labile, for S. faecalis (e.g. PGA+leucovorin + tetrahydroPGA)

9. Oxygen-labile, for $S$. faecalis (e.g. tetra- $\quad(24 \cdot 8$ less $11 \cdot 2)=13 \cdot 6 \dagger \quad(22 \cdot 3$ less 10.1) $=12 \cdot 2 \dagger$ hydroPGA)

* Experimentally determined value (see Table 2).

The values presented in Table 3, it may be noted, agree very well with theoretical calculations. For example, the oxygen-stable fraction stimulatory for Pediococcus cereviseae +the oxygen-labile fraction stimulatory for $\boldsymbol{P}$. cereviseae + the oxygenstable fraction stimulatory for Streptococcus faecalis but not for $P$. cereviseae, should 
be equivalent to the sum total of all the folic acid activity present in the sample. This value was determined experimentally by assay with $\boldsymbol{S}$. faecalis, under anaerobic conditions, to be 24.8 or $22.3 \mu \mathrm{g}$. $/ \mathrm{ml}$. of spent medium (Table 2 ). This can be determined by the following scheme:

\author{
Oxygen-stable fraction \\ Oxygen-labile fraction \\ Oxygen-stable fraction (stimulatory \\ for $S$. faecalis but not for \\ P. cereviseae)
}

Total activity of sample on a theoretical basis

$\begin{array}{cc}\text { Sample no. 1 } & \text { Sample no. 2 } \\ (\mu \mathrm{g} . / \mathrm{ml} .) & (\mu \mathrm{g} . / \mathrm{ml} .) \\ 3.7 & \mathbf{2 \cdot 9} \\ 11 \cdot 1 & \mathbf{8 \cdot 7} \\ 7 \cdot 5 & 7 \cdot 2 \\ & \\ & \\ \mathbf{2 2 . 3} & 18 \cdot 8\end{array}$

It may be noted that the values for the oxygen-labile fraction for $\boldsymbol{P}$. cereviseae and the oxygen-labile fraction for $S$. faecalis (Table 3 ) are nearly equal, which would indicate that the same chemical entities, e.g. tetrahydroPGA, are being assayed Since there appears to be little difference between values for the oxygen-stable fraction determined by assay with Lactobacillus casei (but having no activity for $\boldsymbol{P}$. cereviseae) and the oxygen-stable fraction for $\boldsymbol{S}$. faecalis (having no activity for $\boldsymbol{P}$. cereviseae), it may be concluded that here again the same chemical entities, e.g. PGA, are being assayed.

From these observations, it would appear that three kinds of folic acid compounds are present in $24 \mathrm{hr}$. spent medium of Lactobacillus plantarum: (1) a fraction which is oxygen-stable and supports the growth of Pediococcus cereviseae (such as leucovorin, leucovorin-anhydride, or ${ }^{10} \mathrm{~N}$-formyltetrahydroPGA); (2) a fraction which is oxygen-labile and supports the growth of $\boldsymbol{P}$. cereviseae (such as tetrahydroPGA or dihydroPGA); (3) a fraction which is oxygen-stable and does not support the growth of $\boldsymbol{P}$. cereviseae but which is active for Streptococcus faecalis and Lactobacillus casei (such as PGA, ${ }^{10} \mathrm{~N}$-formylPGA, folylglutamate, thymine, thymidine, ${ }^{10} \mathrm{~N}$ formyldihydroPGA). It would also appear that pteroic acid and ${ }^{10} \mathrm{~N}$-formylpteroic acid are not present, since these compounds are active for $S$. faecalis but not for L. casei.

The foregoing generalizations cannot be considered as proved by the evidence presented, but merely offer a reasonable explanation based on the recent developments concerning folic acid metabolism. As mentioned, much more information needs to be obtained about the effect of $\mathrm{pH}$ value on compounds other than tetrahydroPGA when the response is measured with reference to leucovorin standard curves. In addition, more data are needed about the quantitative response of different assay organisms to these compounds when interpreted in terms of the response of the assay organisms to leucovorin.

The elimination of phenylalanine, as being identical with the factor synthesized by Streptococcus faecalis, differs from the observation reported by Judge (1958). However, since Judge used filtrates of cultures which had been concentrated in vacuo 5- to 10-fold, and since these materials were applied to chromatograph paper in a single 'spot' in 5-10 $\mu \mathrm{l}$. quantities, it is quite possible that interference with the migration of amino acids occurred in a manner similar to that found here for shikimic acid, namely, because of a large concentration of other solutes in the concen- 
trate. Since ninhydrin was used as the indicator, all $\alpha$-amino acids should have been detected. The system used in the present investigation used a biological indicator, the growth of Lactobacillus plantarum, the other member of the syntrophic pair, which specifically indicated the position of the phenylalanine-active factor on the agar plate.

The demonstration that neither phenylalanine nor shikimic acid was identical with the factor synthesized by Streptococcus faecalis and responsible for supporting the growth of Lactobacillus plantarum, suggests the possibility that the factor may be one of the compounds which have been suggested or demonstrated as intermediates in the biosynthesis of phenylalanine. Of such compounds $(a)$ tyrosine cannot replace the phenylalanine requirement of $L$. plantarum, and (b) quinic acid and phenyl pyruvic acid cannot replace the phenylalanine requirement of $L$. plantarum (Nurmikko, 1954). Other possibilities about the nature of the substance involved are 5-dehydroshikimic acid, 5-dehydroquinic acid and shikimic acid-5phosphate; these, however, were not explored.

This work was aided by a contract between the Office of Naval Research (Department of the Navy) and the University of Maryland, NR 103-455, and by a National Science Foundation Summer Fellowship, and is taken from a thesis submitted to the Graduate School of the University of Maryland, by N. R. Kreig, Jun., in partial fulfilment of the requirements for the degree of Doctor of Philosophy. The authors wish to express their thanks to Dr T. Shiota (National Institute of Dental Research, National Institutes of Health, Bethesda, Maryland, U.S.A.) for valuable suggestions as well as for samples of some of the materials used in this work.

\section{REFERENCES}

Block, R. J., Durrum, E. L. \& Zweig, G. (1958). A Manual of Paper Chromatography and Paper Electrophoresis. 2nd ed. New York: Academic Press.

Difco Manual of Dehydrated Culture Media and Reagents for Microbiological and Clinical Laboratory Procedures (1953). 9th ed. Detroit: Difco Laboratories, Inc.

Donaldson, K. O. \& KereszTesy, J. C. (1959). Naturally occurring forms of folic acid. I. 'Prefolic A': preparation of concentrate and enzymatic conversion to citrovorum factor. J. biol. Chem. 234, 3235.

Judge, L. F. (1958). Nutritional Symbiosis Through Microbial Associations. Ph.D. thesis, University of Maryland.

KoFT, B. \& Morrison, J. H. (1956). Symbiotic biosynthesis of folic acid-like growth factors. J. Bact. 72, 705.

KrIEg, N. R. \& Pelczar, Jun., M. J. (1960). Delayed utilization of pteroylglutamic acid by Lactobacillus plantarum. J. Bact. 80, 242.

LEwis, J. C. (1942). A lactobacillus assay method for $p$-aminobenzoic acid. J. biol. Chem. 146, 441 .

NuRmIKko, V. (1954). Symbiosis experiments concerning the production and biosynthesis of certain amino acids and vitamins in associations of lactic acid bacteria. Ann. Acad. Sci. fenn. Ser. A, sec. 2, 54, 7.

SaUberlich, H. E. (1949). The effect of folic acid upon the urinary excretion of the growth factor required by Leuconostoc citrovorum. J. biol. Chem. 181, 467 . 